\title{
The Pierre Auger Observatory
}

\author{
Maria Teresa Dova ${ }^{\text {a*}}$, for the Pierre Auger Observatory Collaboration ${ }^{\dagger}$ \\ a Universidad Nacional de La Plata, C.C. 67, 1900 La Plata, Argentina
}

One of the foremost issues in astrophysics today is that of the origin of the ultra high energy cosmic rays. The Pierre Auger Observatory (PAO), currently under construction in the Province of Mendoza, Argentina, is a broadly based international effort to make a high statistic study of the upper-end of the cosmic ray spectrum. The PAO is the first experiment designed to work in a hybrid detection mode. It consists of an array of 1600 particle detectors spread over $3000 \mathrm{~km}^{2}$ and four fluorescence telescopes placed on the boundaries of the surface array. The concept of the experiment as well as the current status and future prospects are presented.

\section{Why bother with a new cosmic ray ex- periment?}

A key issue to be considered in the search for the origin of extremely high energy cosmic rays is the opacity of the microwave background radiation to the propagation of ultra high energy cosmic rays (UHECR) which yields the well known effect called the GZK cutoff. The first treatments [1] indicated a sharp cutoff for cosmic rays with energies above $5 \times 10^{19} \mathrm{eV}$ due to the process $\gamma+p \rightarrow \Delta \rightarrow p / n \pi$. A similar phenomenon of energy degradation occurs for nuclei due to process of photodisintegration which is very important in the region of giant resonances, but here the diffuse infrared background is of greater importance than the $2.7 \mathrm{~K}$ radiation. Many calculations have been performed using various techniques to study the modification of the cosmic ray spectrum [2] and the general features are now well stablished.

The puzzle set by the observation of UHECR with energies above $10^{20} \mathrm{eV} \mathrm{[3],} \mathrm{which} \mathrm{may} \mathrm{be} \mathrm{ev-}$ idence of new physics or exotic particles, is nowadays one of the central subjects in high energy astroparticle physics.

The existence of UHECR has motivated many detailed studies concerning the generation of such particles as well as their propagation en route to Earth. A complete discussion of most of the mod-

\footnotetext{
"dova@fisica.unlp.edu.ar

†Pierre Auger Observatory Collaboration list at http://auger.cnrs.fr/collaboration.html
}

els for the production of UHECRs can be found in the reviews $[4,5]$ and references therein. Production mechanisms have been commonly classified into two groups: a) botton-up models, which consider conventional acceleration of UHECR in rapidly evolving processes in known astrophysical objects [6-8] and b) top-down models suggesting that particles are not accelerated but are stable decay products of supermassive particles[4]. Sources of these exotic particles could be topological defects (TD) relics from early universc phase transitions associated with spontaneous symmetry breaking underlying unified models of high energy interactions. A general characteristic of top-down models is that, alongside protons, many photons and neutrinos are also produced giving an extra signature to these processes. Detection of neutrinos would be an important clue for identifying UHECR sources.

Above $10^{15} \mathrm{eV}$ all the measurements are indirect: the high energy particle enters in the atmosphere and interacts with the air molecules initiating a cascade of particles which can be detected either by a surface array of detectors spread over a large area or with large aperture telescopes. This last technique can be used due to the fact that during the development of the extensive air showers (EAS), the charged secondaries excite the nitrogen molecules with a subsequent emission of fluorescence light.

The upper end of the cosmic ray spectrum is shown in Fig.1, with data collected by experi- 


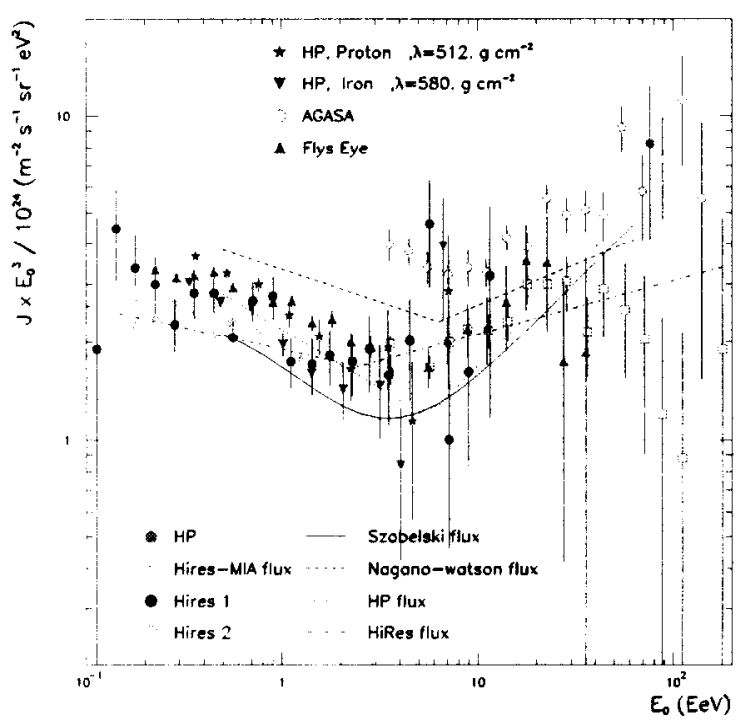

Figure 1. Cosmic ray ray spectrum. The differential flux is multiplied by an energy dependent power $E^{3}$. The compilation is from ref.[14], with data from four experiments: surface arrays (Haverah Park, AGASA) and fluorescence detectors (Fly's Eye, HiRes).

ments using the two techniques mentioned above: $\Lambda \mathrm{G} \Lambda \mathrm{S} \Lambda$ [9], monocular HiRes [10], HiRes-MIA [11], Fly's Eye stereo [12] and the recently reanalysed data from Haverah Park [13]. The figure also includes parameterizations of the energy spectrum. See [14] for details. The spectrum above the so-called "ankle" $\left(5 \times 10^{18} \mathrm{eV}\right)$ is a confused region due to poor statistics, uncertain energy resolution and uncertainties in energy conversion arising from models, lack of knowledge of the mass composition and the fluoresce yield efficiency. The discrepancy between the AGASA and HiRes flux is also evident. It is worth mentioning that the energy calibration is performed in ground array experiments using simulation results and then relies on the assumption of the high energy hadronic interaction model. The re- sults of the AGASA experiment were obtained using the hadronic interaction code QGSJET [15]. On the other hand HiRes relies on the knowledge of fluorescence yield and atmospheric properties. Dedicated laboratory measurements of the fluorescence yield need to be performed to reduce existing errors.

To get information about the cosmic ray origin, studies of anisotropy together with the analysis of the spectrum and composition are mandatory. The present experimental data seem to be consistent with an isotropic distribution of sources, in sharp contrast to the anisotropic distribution of light in the local supercluster. However, there may be clusters. The AGASA experiment has presented an analysis of their extremely high energy events, suggesting indications of clustering on an angular scale of $2.5^{\circ}$ with a probability of chance coincidence of less than $1 \%$. It is remarkable that none of those clusters is on the Galactic plane suggesting that UHECR are most likely extragalactic in origin. A crucial point in the search for the origin of UHECR is to locate their sources. The question is to what extent it is possible to do astronomy with the UHECR detected. Searches for correlations of the observed multiplets with the location of candidate sources or with distribution of astrophysical objects in our neighborhood have been made with negative results. It is evident that the present statistics are not sufficient to make a final conclusion about large scale anisotropies and /or clusters from the analysis of arrival direction distribution.

Another ingredient to consider in the search for the origin of these very energetic particles is the chemical composition of the UHECR detected. In present experiments the interpretation of data, in all cases, depends on the physics of the cascade included in the event generators. There seem to be only some hints about photon fluxes above $10^{19} \mathrm{eV}$ but not much is known about hadronic masses. An analysis of the longitudinal profile of the highest energy event reported by Fly's Eye showed incompatibility with a photon primary [16]. Studies of the data of inclined showers collected by Haverah Park excluded photons at $10^{19} \mathrm{eV}$ at the $40 \%$ level [17]. This result is consistent with the analysis from the muon content of showers reported 
by AGASA [18].

The questions raised by the results concerning spectral shape, anisotropy and composition of ultra high energy cosmic rays clearly indicate the need of greater statistics at the upper-end of the CR spectrum, with accurate measurements of primary energies. The need for new experiments, with full sky coverage and uniform exposure for anisotropy studies, as well as new techniques to determine the composition in a Monte Carlo independent fashion is evident. The Pierre Auger Observatory (PAO), presently under construction in Malargüe, Province of Mendoza in Argentina is the next cosmic ray experiment aimed at giving conclusive answers about the origin and nature of the UHECR.

\section{Concept of Pierre Auger Observatories}

\subsection{Hybrid detectors}

The PAO is designed to measure the energy, arrival direction and primary species with unprecedented statistical precision. The hallmark of the PAO is its unique capability of working in a hybrid detection mode. Particle showers are simultaneously observed by a ground array and by fluorescence detectors. Ground detectors sample the particles of the extensive air shower hitting the ground, while fluorescence detectors follow the development of the shower in the atmosphere by detecting the fluorescence light produce by the interaction of charged secondaries [19].

To obtain full sky coverage two mainly identical observatories will be built, one in the Southern hemisphere and another in the Northern hemisphere. Construction of the Southern Observatory began in 2000 at the foot of the Andes in the middle west of Argentina. This site is specially interesting since from it, the centre of the Galaxy is visible.

The size of each Observatory is chosen to collect high statistics above the expected GZK cutoff, with 1600 particle detectors covering an area of $3000 \mathrm{~km}^{2}$, overlooked by four fluorescence detectors. The surface array stations are water Čerenkov detectors. Figure 2 shows the layout of the Argentinian site with the position of the surface array detectors and the fluorescence eyes at

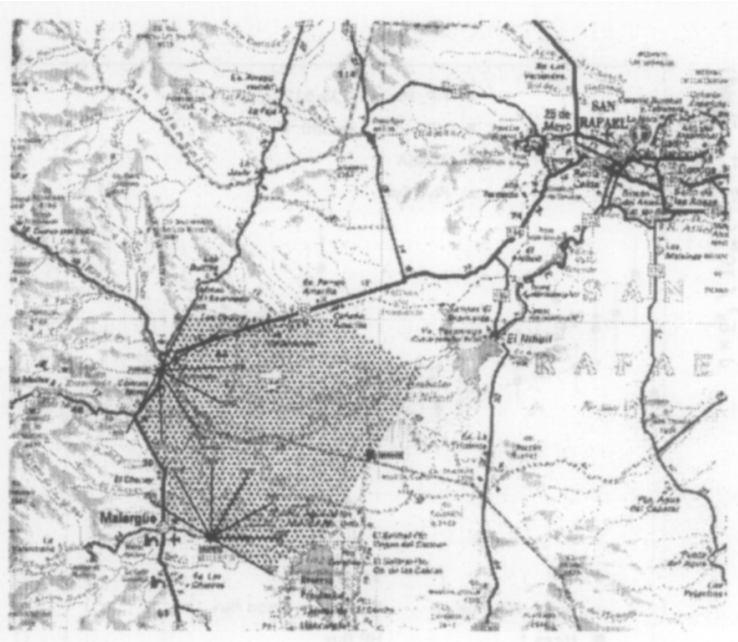

Figure 2. Layout of the southern Pierre Auger Observatory. Surface detectors will be installed in the dotted area and four fluorescence detectors at the periphery of the array

the periphery (Cerro Los Leones, Coihueco, Los Morados and the northern eye). The lines define the azimuth acceptance of the first two fluorescence detectors that will become operational.

The water Cerenkov detection technique and the fluorescence technique, both used in previous experiments, working together are the most powerful instruments for observing extensive air shower properties and hence the study of UHECR. Approximately $10 \%$ of the showers detected by PAO will be observed by both surface and fluorescence detectors, allowing control of unwanted systematics in the primary energy determination. Thus, the hybrid data set will be used to intercalibrate the detectors, providing confidence in the surface array results alone. The hybrid data set will also provide a distribution function in multidimensional parameter space consisting of the quantities sensitive to the mass composition making it possible to constrain the choice of high energy hadronic interaction models. 


\subsection{Ground Array}

The secondary particles produced by a cosmic ray particle can be detected with stations deployed on a regular grid at the observation level. The aperture of the surface array is well defined and large. The density of particles falls off with the distance to the shower core and this can be parameterized by a lateral distribution function (LDF), which, of course, depends on the characteristics of the detectors used. Fig 3 (left) shows a simulated LDF of $\gamma$, electrons and muons at ground level for a $10^{19} \mathrm{eV}$ proton shower, as well as the corresponding distributions convolved with the response of a typical PAO water Čerenkov detector (right). The particle density at a large distance from the shower core is commonly used as an energy estimator with the conversion factor evaluated by sinulations. See ref. [20] for general details about the techniques.
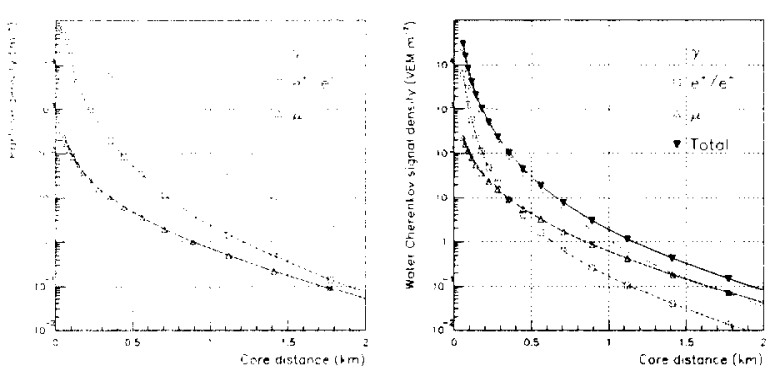

Figure 3. Left: Particle density distributions as a function of the distance from the core. Right: The particles have been convolved with the response of a $1.2 \mathrm{~m}$ deep water Čerenkov detector.

An initial estimate of the shower direction is obtained from the relative arrival times of signal at a minimum of 3 non-collinear detectors, treating the shower front as if it were planar. Muons in the EAS have higher energies than electromagnetic particles, which in addition suffer significant scattering and energy loss. Thus, the muonic component tends to arrive earlier and over a shorter period of time than the electromagnetic one. The large area of the detectors enables the early muons to be collected in sufficient number to allow accurate timing of the arrival of the shower front. These signatures may also help to distinguish $\mu$ s from electrons and $\gamma \mathrm{s}$ providing a useful tool to determine the primary composition. In summary, besides the lateral distributions of signals, there are additional observables which correlate with the position of maximun development of the shower and hence to primary particle type, such as the arrival time profile of shower particles, the steepness of the LDF and the shower front curvature.

The ground array of the Pierre Auger Observatories consists of 1600 stations spaced by 1.5 $\mathrm{km}$. Each detector is a cylindrical, opaque tank of $10 \mathrm{~m}^{2}$ and a water depth of $1.2 \mathrm{~m}$, where particles produce light by Cerenkov radiation. The filtered water is contained in an internal coating which diffusely reflects the light collected by three photomultipliers (PMTs) installed on the top. The large diameter PMTs $(\approx 20 \mathrm{~cm})$ hemispherical photomultiplier are mounted facing down and look at the water through sealed polyethylene windows that are integral part of the internal liner. The signals are processed locally and a second level trigger is identified before transmitting the data to the central acquisition system [21]. Water tanks allow the detection of the very numerous photons present in showers. In addition, the depth enables showers to be detected efficiently over a wide angular range. Due to the size of the array the stations have to work in an autonomous way. Thus the stations operate on battery-backed solar power and communicate with a central station by using wireless LAN radio links [22]. The time information is obtained from the Global Positioning Satellite (GPS) system [23]. Figure 4 shows a water Cerenkov detector installed in the Southern Observatory. Mounted on top of the tank are the solar panel, electronic enclosure, mast, radio antenna and GPS antenna for absolute and relative timing. A battery is contained in a box attached to the tank.

The expected angular resolution for the water Cerenkov detector array of the Southern Auger 


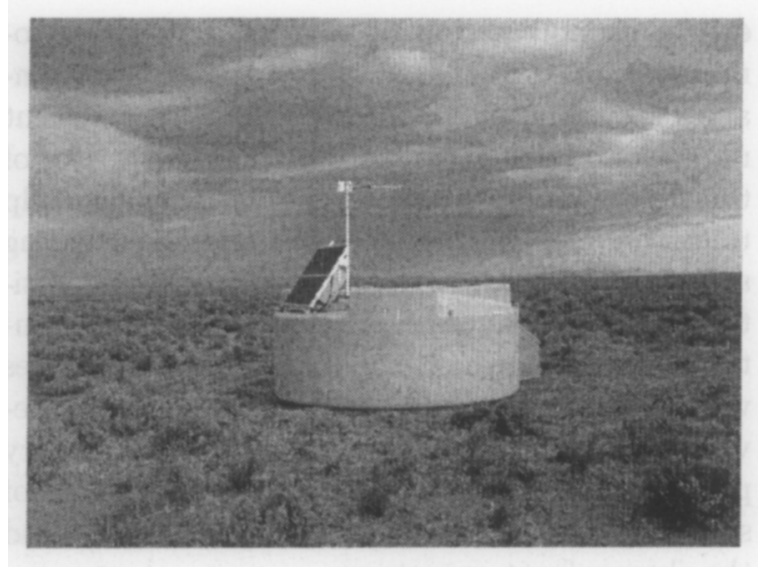

Figure 4. A typical surface detector is shown in this figure. See the text for the description of the components of the station.

Observatory is less than $1^{\circ}$ for all energies improving substantially with increasing zenith angle up to $60^{\circ}$ and improving for large events above $10^{20} \mathrm{eV}$. The expected energy resolution is estimated to be $12 \%$, averaged over all energies (assuming a proton-iron primary mixture), falling to $10 \%$ at $10^{20} \mathrm{eV}$. The limiting aperture for the full Southern Observatory array and for zenith angle less than $60^{\circ}$ is $7350 \mathrm{~km}^{2} \mathrm{sr}$. The detection effciency at the trigger level should reach $100 \%$ for energies above $10^{19} \mathrm{eV}$ [24]. Additionally, if events above 60 degrees can be analyzed effectively, the aperture will increase by about $50 \%$.

\subsection{Fluorescence detectors and the bene- fits of the hybrid mode}

The Auger fluorescence detectors (FD) are expected to be operated always in conjunction with the SD. The fluorescence technique provides the most effective way to measure the energy of the primary particle. The amount of fluorescence light emitted is proportional to the number of charged particles in the showers allowing a direct measurement of the longitudinal development of the EAS in the atmosphere. For this, the sky is viewed by many segmented cycs using photo-

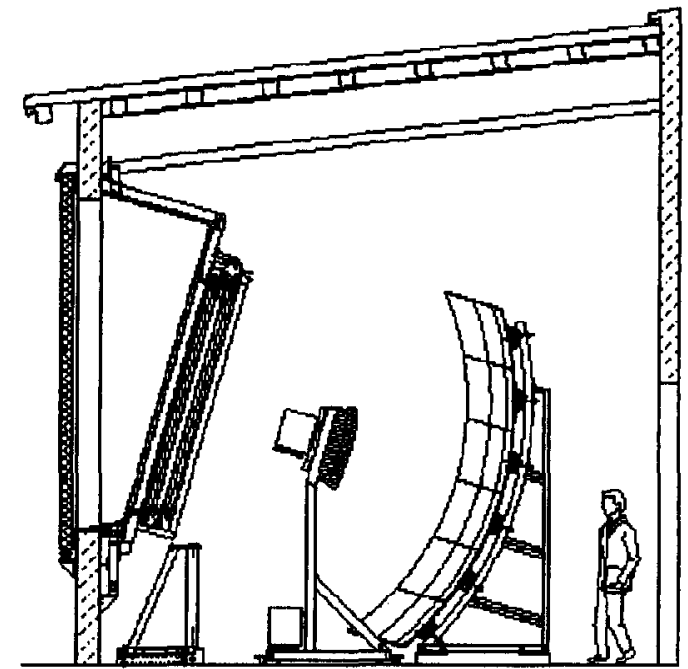

Figure 5. The Auger Fluorescence telescope

multipliers. From the measured shower profile the position of the shower maximum $X_{\max }$ can be obtained. The energy in the electromagnetic component is calculated by integrating the measured shower profile. Corrections for atmospheric attenuation of the fluorescence light and contamination of the signal by Cerenkov light have to bc made, using monitoring details at the observatory [25].

A good shower reconstruction by means of the FD $\left(X_{\max }\right.$ resolution $\left.\approx 20 \mathrm{~g} \mathrm{~cm}^{-2}\right)$ depends on the ability to determine the position of the shower axis inside the shower-detector plane (the plane in space containing the shower axis and a point representing the detector). This is done using the light arrival times in each pixel [26]. However, a better determination of the shower axis is achieved if the shower is observed in the hybrid mode, using timing information from the SD [27]. Good determination of the shower axis is 
mandatory for good energy and mass composition assignments.

Four fluorescence detectors will be installed at the periphery of the ground array. This arrangement reduces the dependence on a precise knowledge of light transmission through the atmosphere. For the design of the fluorescence telescopes the principle of a wide Schmidt camera is used. Figure 5 shows the main components of a fluorescence eye: a large spherical mirror with a radius of curvature of $3.4 \mathrm{~m}$, a pixel camera with 440 PMTs in the focal surface and a diaphragm with an entrance glass window. This filter allows reduction of night background with respect to the fluorescence signal and also serves to protect the equipment from dust. Each fluorescence detector will be housed in a single building. A picture of the FD building at Los Leones taken from the 50 metre concentrator tower for data transmission is shown in Figure 6.

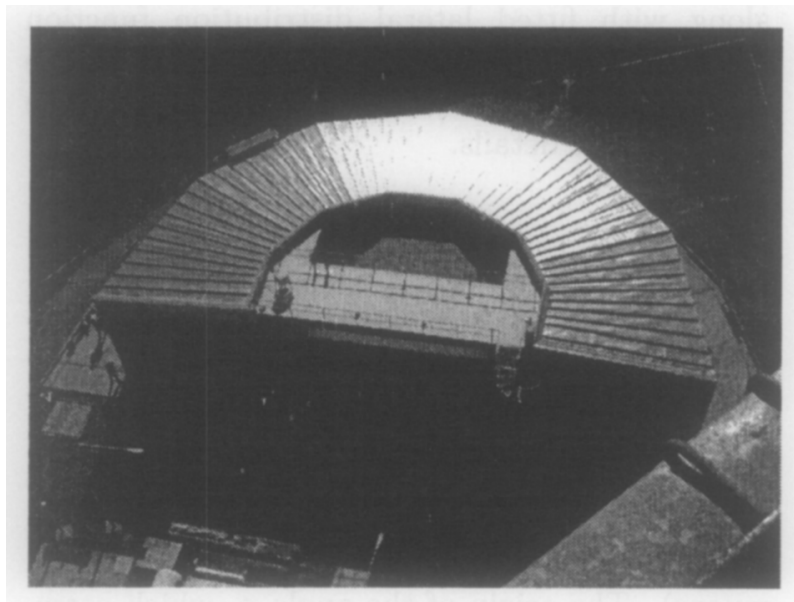

Figure 6. A photo of the building for the fluorescence detectors at Los Leones site (south of the surface array)

The first cosmic ray event detected by one of the two prototype telescopes installed at Los Leones is displayed in Figure 7. A twenty pixel track, produced by light from a CR shower, with a length of $8 \mu \mathrm{s}$ can be seen. The angular velocity of the shower image across the sky allows the distance of the shower core to be established as 5 $\mathrm{km}$. The time duration of the signal in the field of view of the telescope corresponds to a track length of $2.4 \mathrm{~km}$. The mirror inverts the picture. Particles from the sky enter from the bottom of the camera.

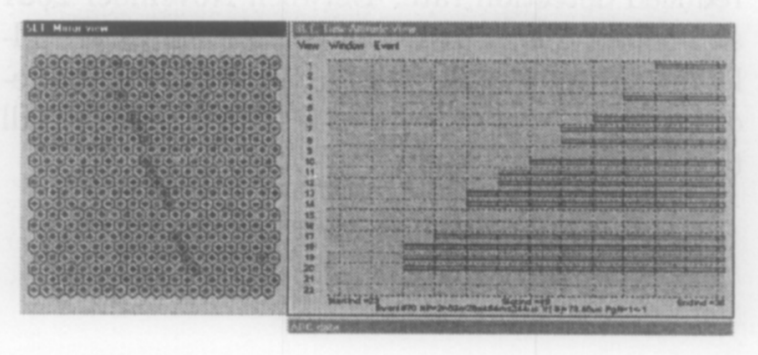

Figure 7. First high energy cosmic ray observed by one of the prototype telescopes at Los Leones. One time slot corresponds to $1 \mu \mathrm{s}$

In the hybrid mode, the Pierre Auger Observatory is expected to have $6 \%$ energy resolution and an angular precision of $0.5^{\circ}$ at $10^{20} \mathrm{eV}$. The detector is optimized for energies above $10^{19} \mathrm{eV}$, but good reconstruction is expected at energies down to $1 \mathrm{EeV}$. The hybrid data set will provide the best evaluation of primary species, allowing a simultaneous fit to all parameters sensitive to mass composition.

\section{Present status and future prospects}

The construction of the Argentinian site started in 2000. An "Engineering Array" (EA) consisting of 40 surface stations and two prototypes fluorescence telescopes was completed and it is operating in steady state since January 2002. The area covered by this small array is approximately $46 \mathrm{~km}^{2}$. In the middle of this array two detectors are located only $11 \mathrm{~m}$ apart. These detectors will allow the direct evaluation of the ac- 
curacy of timing and density measurements. The goal of the EA was to evaluate the performance of every component and system before full production and deployment. The performance of the hybrid operation was successfully demonstrated by simultaneously recording showers by both surface and fluorescence detectors. It is worth mentioning that with this small array, the parameters of the showers can be measured with a precision similar to that expected with the full array, but at reduced detection rate. Between November 2001 and March 2002 about 70 hybrid events were collected. These data will be useful to check the reconstruction and analysis tools, though too small to derive physics results.

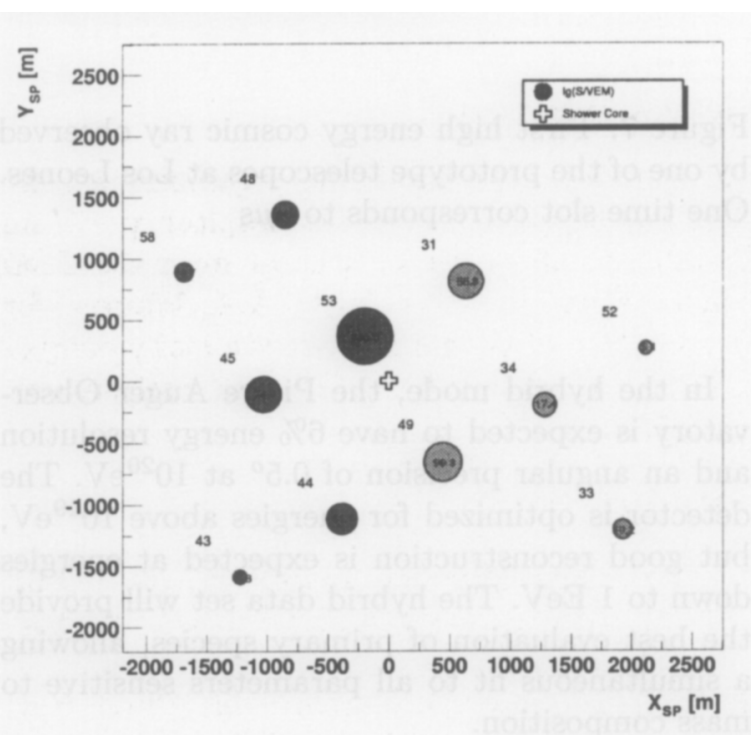

Figure 8. Integrated detector signals in shower plane coordinates. The size of the circles are proportional to $\log (S /[V E M])$. See text for more details.

In what follows some preliminary analysis of events detected with the EA are presented. In

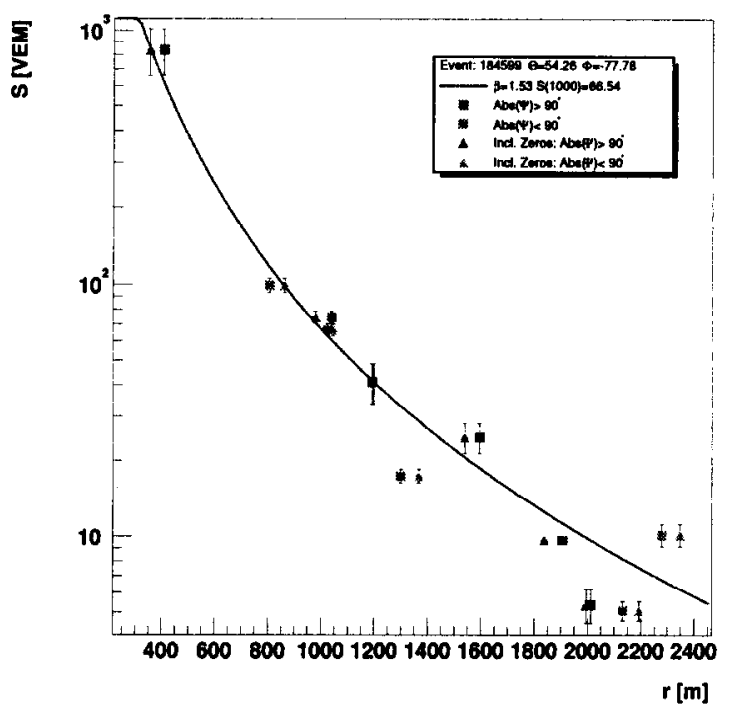

Figure 9. The water Čerenkov signals $\rho(r)$ (dots) along with fitted lateral distribution function (solid line). The error bars correspond to the spread of the signal of the three PMTs. See the text for more details.

late April an event which triggered 11 stations was rccorded [28]. The zenith angle was inferred from the relative arrival times of the shower front at the surface stations and results in $\theta=$ $57.3^{\circ} \pm 0.5^{\circ}$. Figure 8 shows the position of the stations in shower plane coordinates and the integrated signal in units of VEM (vertical equivalent muons). The origin of the angle $\phi$, which corresponds to the direction of the positive $\mathrm{x}$-axis in the rotational plane, reflects the incoming direction of the shower. The size of the circles are proportional to $\log (\mathrm{S} / \mathrm{VEM})$. Detectors such as $58,48,45,53,44$ correspond to late arrival time particles.

The lateral distribution function was parameterized with a NKG-like distribution and is shown in Figure 9. Light grey points indicate detectors with early arrival time and black points stations with late arrival time particles. The signal at 
$1000 \mathrm{~m}$ from the core corresponds to a preliminary energy estimate of $25 \pm 4 \mathrm{EeV}$. A detailed analysis of the signals from the triggered stations was performed. As a result a vast amount of infurmation about the characteristics of the showers that is contained in the tracks recorded by the surface detectors was obtained. The corresponding analysis of the complete data collected by the EA will be presented in the near future.

The geometric reconstruction of a shower with the FD is performed using timing and light information from triggering pixels. The ADC trace in each pixel allows accurate determination of the time at which the shower front passed the centre of the pixel. After calibration, the FADC traces are converted to light fluxes seen by the pixels, in units of $379 \mathrm{~nm}$-equivalent photons reaching the mirror diaphragm per 100 ns time bin. Once the shower axis is determined, the number of charged particles as a function of atmospheric depth can be obtained. The corresponding longitudinal profile is finally derived after corrections, as described in section 2.3. Information on the atmospheric transparency is extremely important. A dedicated LIDAR system has been built to perform on-line monitoring of the atmospheric parameters. The laser backscattering method is used to parametrize the attenuation length over the detection volume [29].

In Figure 10, preliminary results of a profile reconstruction, using the $\mathrm{FD}$ is shown. The position of depth of maximum is derived by fitting a Gaiser-Hillas function to the observed longitudinal profile. The corresponding value of $X_{\max }$ is $670 \mathrm{~g} \mathrm{~cm}^{-2}$. The preliminary primary energy derived using only the FD, which depends on the fluorescence yield measurements as well as light scattering and absorption in the atmosphere, is $1.3 \times 10^{19} \mathrm{eV}$.

\section{Conclusions}

The Pierre Auger Observatory will be a powerful tool for the study of the UHECR. Using a small engineering array, all sub-systems of the Observatory have been shown to work at, or above, specification. The Observatory will be completed during 2005 .

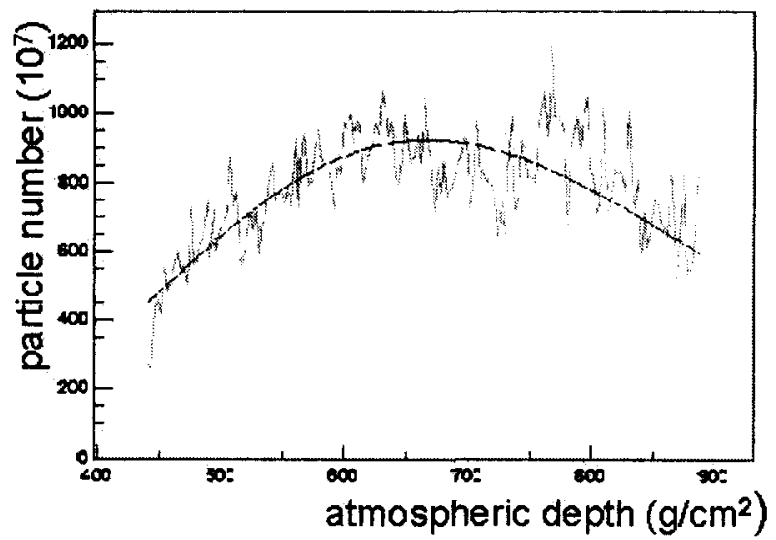

Figure 10. Reconstructed shower longitudinal profile.

\section{Acknowledgments}

I would like to thank the organizers for the financial support and warm hospitality. I am grateful to all of my colleagues from the PAO collaboration, in particular to A.A. Watson, for illuminating discussions about the many components of the observatory and data analysis presented here.

\section{REFERENCES}

1. K. Greisen, Phys Rev Letters 16, 748 (1966), G.T.Zatsepin and V.A.Kuzmin, JETP Lett.4, 78 (1966)

2. C.T. Hill and D.N. Schramm, Phys. Rev. D 31, 5648 (1985); V.S.Berezinsky and S.I. Grigoreva, Astron. Astrophys.199, 1(1988); J.L. Puget, F.W. Stecker and J.H. Bredekamp, Astrophys. J, 205, 638 (1976); S. Yoshida and M. Teshima, Prog.Theor.Phys.89, 833 (1993); L.A. Anchordoqui, M.T.Dova, L.N.Epele and J. Swain, Phys. Rev. D 55, 7356 (1997); L.A. Anchordoqui, M.T.Dova, L.N.Epele and J. Swain, Phys. Rev. D 57, 7103 (1998); L.N. Epele and E. Roulet, JHEP 9810, 009 (1998); T. Stanev, R. Engel, A. Mucke, R. Protheroe 
and J.P. Rachen, Phys. Rev. D 62,093005 (2000).

3. J. Linsley, Phys. Rev. Lett. 10, 146 (1963); M.A. Lawrence, R.J.O. Reid, A.A. Watson, J.Phys G. 17, 733 (1991); N. Hayashida et al, Phys. Rev. Lett. 74, 3491 (1994); D.J.Bird , et al, Astrophys. J. 441, 144 (1995); N. Sakakiet al [AGASA Coll.] Proc. of 27th ICRC (Hamburg) 1, 333 (2001)

4. P. Bhattacharjee and G. Sigl, Phys. Rep. 327, 2000

5. A.V. Olinto, Phys. Rep. 333-334, 329 (2000)

6. M. Hillas, Ann.Rev.Astron.Astrophys. 22, 425 (1984); P.L. Biermann, J.Phys.G (Nucl.Part.Phys) 23, 1 (1997).

7. R.D. Blanford, Phys.Scripta, T85, 191 (2000).

8. J.P. Rachen and P.L. Biermann, Astron. Astrophys 272, 161 (1993).

9. N. Hayashida et al, Astrophysics J. 522, 225 (1999),

10. C.C.H. Jui [HiRes Coll.] Proc. of 27th ICRC (Hamburg) 1, 354 (2001)

11. T. Abu-Zayyad et al [HiRes-MIA Coll.] Astrophys. J. 557, 686 (2001).

12. D. J. Bird et al, Ap. J. 424, 491 (1994).

13. M. Ave, J. Knapp, J. Lloyd-Evans, M. Marchesini and A. A. Watson, arXiv:astroph/0112253.

14. A. A. Watson, arXiv:astroph/0112474.

15. N.N. Kalmykov, S. S. Ostapchenko, Yad. Fiz. 56, 105 (1993); N.N. Kalmykov, S. S. Ostapchenko, Bull. Russ. Acad. Sci. (Physics) 58,1966 (1994).

16. F. Halzen et al, Astroparticle Physics 3151 (1995)

17. M. Ave et al, Phys. Rev. Lett. 85, 2244 (2000)

18. K. Shinozaki et al [AGASA Coll.] Proc. of 27th ICRC (Hamburg) 1, 346 (2001).

19. Pierre Auger Project Design Report, 1997, Auger Coll., Fermi National Accelerator Laboratory. (www.auger.org/admin)

20. M. Nagano and A.A. Watson, Rev.Mod.Phys 72,689 (2000).

21. T. Suomijärvi [Pierre Auger Coll.], Proc. of 27th ICRC (Hamburg), (2001).

22. P.D.J. Clark and D. Nitz, [Pierre Auger Coll.], Proc. of 27th ICRC (Hamburg), (2001).

23. C. Pryke et al Nucl. Inst. Methods A354, 354
(1995).

24. M. Ave, J. Lloyd-Evans, A.A. Watson, [Pierre Auger Coll.], Proc. of 27th ICRC (Hamburg), (2001).

25. R. Cester, [Pierre Auger Coll.], Proc. of 27 th ICRC (Hamburg), (2001).

26. H. Gemmeke, [Pierre Auger Coll.], Proc. of 27th ICRC (Hamburg), (2001); H. Gemmeke, M. Kleifges, A. Kopmann, N. Kunka, A. Menshikov, and D. Tcherniakhovski, [Pierre Auger Coll.], Proc. of 27th ICRC (Hamburg), (2001); G. Matthiae [Pierre Auger Coll.], Proc. of 27th ICRC (Hamburg), (2001).

27. B. Dawson and P. Sommers, [Pierre Auger Coll.], Proc. of 27th ICRC (Hamburg), (2001).

28. M. Ave, J. Knapp, M. Marchesini, M. Roth and A.A. Watson, Auger Technical Note GAP-2002-020.

29. A. Filipčič, D. Veberič, D. Zavrtanik, M. Zavrtanik, R. Cester, M. Mostafa, R. Mussa [Pierre Auger Coll.], Proc. of 27th ICRC (Hamburg), (2001). 